\title{
Linear spaces with mixed topology
}

by

A. WIWEGER (Warszawa)

This paper ${ }^{1}$ ) contains a systematic investigation of a topology called mixed topology and determined in a natural way by two given topologies defined in the same linear space $X$. The theory of mixed topologies here presented is closely related to Alexiewicz's investigation of two-norm spaces ([1], [2]) and Orlicz's investigation of Saks spaces ([9], [10]). Roughly speaking, the mixed topology is the unique natural neighbourhood topology corresponding to the sequential topology in a two-norm space. It is also the unique natural extension (to the whole space) of the topology in the unit sphere considered as a Saks space. The connection between spaces with mixed topology and Saks spaces or two-norm spaces makes it possible to apply the theory of linear topological spaces (in particular locally convex spaces) to the investigation of Saks spaces and two-norm spaces. As an example of such applications a theorem on the extension of $\gamma$-linear functionals will be proved (see 2.6.4)

1. Preliminaries. By a linear space we understand any linear space over the field of reals $R$. The restriction to real spaces is not essential and the passage to complex spaces presents no difficulty. If $X$ is a linear space and $a \in X, A \subset X, B \subset X, \alpha \epsilon \mathcal{R}$, then we shall use the following notation:

$$
\begin{aligned}
a+A & =[a+x: x \in A] \\
A \pm B & =[x \pm y: x \in A \text { and } y \in B] \\
\alpha A & =[a x: x \in A]
\end{aligned}
$$

If $y=f(x)$ is a mapping from a set $X$ into another set $Y$ and $Z \subset X$, then the restriction of $f$ to $Z$ is denoted by $f \mid Z$.

Let $X$ be a linear space. If a topology $\tau$ is defined in $X$ in such a way that addition and multiplication by scalars are continuous in both

(1) Presented as a Doctor's Thesis at the Mathematical Institute of the Polish Academy of Sciences on May 9, 1959. 
variables, then $\tau$ is called a linear topology. A linear space $X$ with a linear topology $\tau$ is called a linear topological space and is denoted by $\langle X, \tau\rangle$. If $\tau$ is a linear topology, then $\mathfrak{U}(\tau)$ denotes a basis of neighbourhoods for 0 in the $\tau$-topology. For each $a \in X$ the family of sets $a+U, U_{\epsilon} \mathfrak{U}(\tau)$, is then a basis of neighbourhoods for the element $a$.

If $\tau$ is a linear topology, then there exists a basis $\mathfrak{U}(\tau)$ satisfying the following conditions:

$\left(\mathrm{l}_{1}\right)$ if $U \in \mathfrak{U}(\tau)$ and $\lambda \epsilon \Re, \lambda \neq 0$, then $\lambda U \in \mathfrak{U}(\tau)$,

$\left(1_{2}\right)$ if $U \epsilon \mathfrak{U}(\tau)$ and $\lambda \epsilon \mathcal{R},|\lambda| \leqslant 1$, then $\lambda U \subset U$,

$\left(1_{3}\right)$ if $U \in \mathfrak{U}(\tau)$, then for every $\infty \in X$ there oxists $\lambda \epsilon \%, \lambda \neq 0$, such that $\lambda x \in U$,

$\left(1_{4}\right)$ if $U \in \mathfrak{U}(\tau)$ and $V \in \mathfrak{U}(\tau)$, then thore exists $W \in \mathfrak{U}(\tau)$ such that $W \subset U \cap V$,

$\left(1_{5}\right)$ if $U \in \mathfrak{U}(\tau)$, then there exists $V \in \mathfrak{U}(\tau)$ such that $V+V \subset V$.

In the sequel we shall always suppose that any basis of neighbourhoods under consideration satisfies all the conditions $\left(l_{1}\right)-\left(l_{5}\right)$.

If, conversely, $\mathfrak{U}$ is a family of subsets of $X$ satisfying conditions $\left(\mathrm{I}_{1}\right)-\left(\mathrm{I}_{5}\right)$, then $\mathfrak{U}$ is a basis of neighbourhoods of 0 for some linear topology. A linear topology $\tau$ is called a linear Hausdorff topology if and only if the basis $\mathfrak{U}(\tau)$ satisfies the condition

$\left(1_{6}\right)$ for every $x \in X, x \neq 0$ there exists $U \in \mathfrak{U}(\tau)$ such that $x \notin U$.

A linear topology is called locally convex if and only if there exists a basis of convex neighbourhoods of 0 . Let $\tau_{1}$ and $\tau_{\mathrm{a}}$ be two linear topologies defined on $X$. If for overy $U \epsilon \mathfrak{U}\left(\tau_{1}\right)$ there existis a $V \epsilon \mathfrak{U}\left(\tau_{2}\right)$ such that $V \subset U$, then we say that the topology $\tau_{2}$ is finer than $\tau_{1}$ (or that $\tau_{1}$ is coarser than $\tau_{2}$ ) and we write $\tau_{1} \leqslant \tau_{2}$. If $\tau$ is a linear topology on $X$ and $Z \subset X$, then for each element $a \in Z$ we can take the family of sets $(a+U) \cap Z$, where $U \in \mathfrak{U}(\tau)$, as the basis of neighbourhoods of $a$. The topology thus defined in $Z$ is denoted by $\tau \mid Z$ and called topology induced on $Z$ by $\tau$.

If $\left\langle X, \tau_{1}\right\rangle$ and $\left\langle Y, \tau_{2}\right\rangle$ are (not necessarily linear) topological spaces, and if $y=u(x)$ is a continuous operation from $X$ to $X$, then wo say that the operation $u$ is $\left(\tau_{1}, \tau_{2}\right)$-continuous. If in particular $\left\langle X, \tau_{1}\right\rangle$ and $\left\langle Y, \tau_{2}\right\rangle$ are linear topological spaces, then instead of " $u$ is distributive and $\left(\tau_{1}, \tau_{2}\right)$. -continuous" we say " $u$ is $\left(\tau_{1}, \tau_{2}\right)$-linear".

Let $X$ be a linear space: We say that a set $A . X$ absorbs a set $B C X$ if there exists a $\lambda>0$ such that $\lambda B C A$. A subset $B$ of a linear topological space $\langle X, \tau\rangle$ is said to be bounded (or $\tau$-bounded) if $B$ is $a b$ sorbed by every neighbourhood of 0 . The class of all $\tau$-bounded sets is denoted by $\mathrm{Bd}(\tau)$. A set $B$ is $\tau$-bounded if and only if for every sequence $\left\{x_{n}\right\}$ of elements of $B$ the conditions $\lambda_{n} \geqslant 0, \lambda_{n} \rightarrow 0$ imply $\lambda_{n} x_{n} \rightarrow 0$ in the $\tau$-topology.

Let $\langle X, \tau\rangle$ be a locally convex linear Hausdorff topological space. A set $A \subset X$ is called symmetric if $x \in A$ implies $-x \in A$. A set $A \subset X$ is called absorbing if, for every $x \in X$, there is a $\lambda>0$ such that $\lambda x \in A$. A set $A$ is called a barrel if $A$ is convex, symmetric, absorbing and closed. The space $\langle X, \tau\rangle$ is called a $t$-space (espace tonnelé) if all barrels are neighbourhoods of 0 ([5], [6]). The space $\langle X, \tau\rangle$ is called bornological if any convex symmetric set in $X$ which absorbs all bounded subsets of $X$ is a neighbourhood of 0 (see [6]). It is known that

(*) A locally convex linear Hausdorff topological space $\langle\bar{X}, \tau\rangle$ is bornological if and only if, for every locally convex topology $\tau_{1}$ defined on $X$, the condition $\mathrm{Bd}(\tau)=\mathrm{Bd}\left(\tau_{1}\right)$ implies $\tau_{1} \leqslant \tau$.

2.1. Suppose that in a linear space $X$ two linear Hausdorff topologies $\tau$ and $\tau^{*}$ are defined. Let $\mathfrak{U}(\tau)$ and $\mathfrak{U}\left(\tau^{*}\right)$ be bases of neighbourhoods for 0 in topologies $\tau$ and $\tau^{*}$ respectively. Neighbourhoods in $\mathfrak{U}(\tau)$ will be denoted by $U, V, \ldots$, and neighbourhoods in $\mathfrak{U}\left(\tau^{*}\right)$ will be denoted by $U^{*}, V^{*}, \ldots$ In the sequel we shall sometimes postulate (but only when explicitly stated) the following conditions:

(n) $\tau^{*} \leqslant \tau$;

(o) the neighbourhoods belonging to $\mathfrak{U}(\tau)$ are $\tau$-bounded;

(d) the neighbourhoods belonging to $\mathfrak{U}(\tau)$ are convex and $\tau^{*}$-closed.

Condition (0) implies the metrisability of space $\langle X, \tau\rangle$. The space $\langle X, \tau\rangle$ is then a Fréchet space or an incomplete Fréchet space. If the topology $\tau$ is locally convex (and, in particular, if condition (d) is satisfied), then condition ( 0 ) is equivalent to the statement that the space $\langle X, \tau\rangle$ is a normed space (i. e. a $B^{*}$-space).

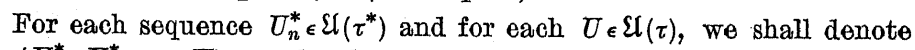
by $\gamma\left(U_{1}^{*}, U_{2}^{*}, \ldots ; U\right)$, or shortly by $U^{r}$, the set

$$
\bigcup_{n=1}^{\infty}\left(U_{1}^{*} \frown U+U_{2}^{*} \cap 2 U+\ldots+U_{n}^{*} \frown n U\right),
$$

i. e. the set of all sums $x_{1}+x_{2}+\ldots+x_{\eta}(n=1,2, \ldots)$, where $x_{k} \in U_{k}^{*}$ and $\frac{1}{k} x_{k} \in U$.

It is easy to verify that the family $\Omega$ of all the sets (1) is a basis of neighbourhoods for 0 in a linear Hausdorff topology. In fact, if $\gamma\left(U_{1}^{*}, U_{2}^{*}, \ldots ; U\right) \epsilon \Re$ and $\lambda \epsilon \mathscr{R}$, then $\lambda \cdot \gamma\left(U_{1}^{*}, U_{2}^{*}, \ldots ; U\right)=\gamma\left(\lambda U_{1}^{*}, \lambda U_{2}^{*}\right.$, $\ldots ; \lambda U)$. Therefore conditions $\left(l_{1}\right)$ and $\left(l_{2}\right)$ are satisfied. For each $x \in \mathcal{X}$ there exists a $\lambda \in \mathcal{R}, \lambda \neq 0$, such that $\lambda x \in U_{1}^{*}$ and $\lambda x \in U$. Then $\lambda x \in \gamma\left(U_{1}^{*}\right.$, Studia Mathematica $\mathrm{xx}$ 
$\left.U_{2}^{*}, \ldots ; U\right)$, which proves $\left(\mathrm{l}_{3}\right)$. If $W_{k}^{*} \subset U_{k}^{*} \cap V_{k}^{*}(k=1,2, \ldots)$ and $W \subset U \cap V$, then $\gamma\left(W_{1}^{*}, W_{2}^{*}, \ldots ; W\right) \subset \gamma\left(U_{1}^{*}, U_{2}^{* *}, \ldots ; U\right) \frown \gamma\left(V_{1}^{*}, V_{2}^{*}\right.$, $\ldots ; V)$, which proves $\left(l_{4}\right)$. Condition $\left(l_{5}\right)$ can be verified as follows: Let us choose $V_{n}^{*} \in \mathfrak{U}\left(\tau^{*}\right)$ and $V \in \mathcal{U}(\tau)$ in such a way that $V_{n}^{*}+V_{n}^{*} \subset U_{n}^{*}$ and $V+V \subset U$. Then $V^{\gamma}+V^{\gamma} \subset U^{\gamma}$, where $V^{\gamma}=\gamma\left(V_{1}^{*}, V_{2}^{*}, \ldots ; V\right)$. In faot, if $x \in V^{\nu}+V^{\nu}$, then $x=y+z$, where $y=y_{1}+y_{2}+\ldots+y_{m}, \quad y_{k} \in V_{k}^{*}$, $\frac{1}{2} y_{k} \in V \quad(k=1,2, \ldots, m)$ and $\quad z=z_{1}+z_{2}+\ldots+z_{n}, \quad z_{k} \in V_{k}^{*}, \quad \frac{1}{k_{k}} z_{k} \in V$ $(k=1,2, \ldots, n)$. Hence (if, for instance, $m \leqslant n) x=\left(y_{1}+z_{1}\right)+\left(y_{2}+z_{2}\right)+$ $+\ldots+\left(y_{m}+z_{m}\right)+z_{m+1}+\ldots+z_{n}$ and it follows from $y_{k}+z_{k} \in U_{k}^{*}$, $\frac{1}{k}\left(y_{k}+z_{k}\right) \in U(k=1,2, \ldots, m), z_{k} \in U_{k}^{*}, \frac{1}{k} z_{k} \in U(k-m+1, m+2, \ldots, n)$ that $x \in U^{\nu}$.

Hence the family $\Omega$ is a basis of neighbourhoods for 0 in a new linear topology. We shall call this topology the mixed topology $\left(^{2}\right)$ determined by the topologies $\tau$ and $\tau^{*}$.

We denote the mixed topology by $\gamma\left[\tau, \tau^{*}\right]$ or shortly by $\tau^{\gamma}$. The mixed topology satisfies condition $\left(l_{0}\right), i$. $e$. it is a Hausdorff topology. This follows at once from the following statement:

2.1.1. For each $U^{*} \in \mathfrak{U}\left(\tau^{*}\right)$ there exists a $\gamma\left(U_{1}^{*}, U_{2}^{*}, \ldots ; U\right) \in \mathfrak{N}$ such that $\gamma\left(U_{1}^{*}, U_{2}^{*}, \ldots ; U\right) \subset U^{*}$.

In fact, for every $U^{*} \in \mathfrak{U}\left(\tau^{*}\right)$ there exists $U_{1}^{*} \in \mathfrak{U}\left(\tau^{*}\right)$ such that $U_{1}^{*}+U_{1}^{*} \subset U^{*}$. Furthermore, there exists $U_{2}^{*} \in \mathcal{U}\left(\tau^{*}\right)$ such that $U_{2}^{*}+U_{2}^{*}$ C $U_{1}^{*}$. By induction, there exists a $U_{n}^{*} \in \mathcal{U}\left(\tau^{*}\right)$ such that $U_{n}^{*}+U_{n}^{*} \subset U_{n-1}^{*}$. We have $U_{1}^{*}+U_{2}^{*}+\ldots+U_{n}^{*} \subset U^{*}$ for each $n$, and therefore $\gamma\left(U_{1}^{*}, U_{2}^{*}\right.$, $\ldots ; U) \subset U^{*}$ for each $U$.

Lemma 2.1.1. may be written in the form

(2)

$$
\tau^{*} \leqslant \gamma\left[\tau, \tau^{*}\right] \text {. }
$$

If condition (n) is satisfied, then

$$
\gamma\left[\tau, \tau^{*}\right] \leqslant \tau .
$$

In fact, for every $U^{\gamma}$ of form (1) there exists a $V \in \mathfrak{U}(\tau)$ such that $V \subset U_{1}^{*} \cap U \subset U^{\nu}$.

$$
\text { If } \tau^{*} \geqslant \tau \text {, then }
$$

$$
\gamma\left[\tau, \tau^{*}\right]=\tau^{*} .
$$

In fact, for every $U^{\gamma}$ there exists a $U^{*}{ }_{\epsilon} \mathfrak{L l}\left(\tau^{*}\right)$ such that $U^{*} \mathrm{C}$ $U_{1}^{*} \cap U \subset U^{\gamma}$. Hence $\tau^{*} \geqslant \gamma\left[\tau, \tau^{*}\right]$, which, together with (2), proves (4).

(2) The term "the space with mixed topology" was first used by A. Alexiewioz and $\mathrm{Z}$. Semadeni in paper [3].
If the topologies $\tau$ and $\tau^{*}$ are locally convex, then the topology $\tau^{\gamma}$ is also locally convex. In fact, if $U_{n}^{*}(n=1,2, \ldots)$ and $U$ are convex then the sets $U_{1}^{*} \cap U+U_{2}^{*} \cap 2 U+\ldots+U_{n}^{*} \cap n U$ are convex, as algebraic sums of convex sets. Therefore the set $\gamma\left(U_{1}^{*}, U_{2}^{*}, \ldots ; U\right)$ is convex as the set-theoretical union of an increasing sequence of convex sets.

Remark. We could take as a basis of neighbourhoods of 0 in the mixed topology the class of all sets of the form

$$
\bigcup_{n=1}^{\infty}\left(U_{1}^{*} \frown \alpha_{1} U+U_{2}^{*} \frown \alpha_{2} U+\ldots+U_{n}^{*} \frown a_{n} U\right),
$$

where $\left\{a_{n}\right\}$ is an arbitrary fixed sequence of real numbers tending to infinity. In fact, there exists a subsequence $\left\{\alpha_{m_{n}}\right\}$ such that $\left|a_{m_{n}}\right| \geqslant n$. Then

and

$$
U_{m_{n}}^{*} \frown n U \subset U_{m_{n}}^{*} \frown \alpha_{m_{n}} U
$$

$$
\gamma\left(U_{m_{1}}^{*}, U_{m_{2}}^{*}, \ldots ; U\right) \subset \bigcup_{n=1}^{\infty}\left(U_{1}^{*} \frown \alpha_{1} U+U_{2}^{*} \frown \alpha_{2} U+\ldots+U_{n}^{*} \frown \alpha_{n} U\right) .
$$

Conversely, if $\left\{k_{n}\right\}$ is an increasing sequence of positive integers such that $k_{n} \geqslant\left|\alpha_{n}\right|$, then $U_{k_{n}}^{*} \cap \alpha_{n} U \subset U_{k_{n}}^{*} \cap k_{n} U$, and

$$
\bigcup_{n=1}^{\infty}\left(U_{k_{1}}^{*} \frown \alpha_{1} U+U_{k_{2}}^{*} \frown \alpha_{2} U+\ldots+U_{k_{n}}^{*} \frown \alpha_{n} U\right) \subset \gamma\left(U_{1}^{*}, U_{2}^{*}, \ldots ; U\right) .
$$

Therefore, the bases of neighbourhoods of the form (1) and (5) are equivalent.

2.2. Let $\tau$ and $\tau^{*}$ be two linear Hausdorff topologies defined on $X$. Let $\tau^{\prime}$ be an arbitrary linear topology defined on $X$. We say that the topology $\tau^{\prime}$ satisfies condition $\left(\mathbf{P}_{1}\right)$ (with respect to the pair $\left(\tau, \tau^{*}\right)$ ) if

$$
\tau^{\prime}\left|Z=\tau^{*}\right| Z \quad \text { for each } Z \in \operatorname{Bd}(\tau) .
$$

2.2.1. The mixed topology $\gamma\left[\tau, \tau^{*}\right]$ satisfies the condition $\left(\mathbf{P}_{1}\right)$.

2.2.2. If the topology $\tau$ satisfies condition (0), then for every linear topology $\tau^{\prime}$ defined on $X$, the condition

$$
\tau^{\prime}\left|Z \leqslant \tau^{*}\right| Z \quad \text { for each } Z \in \operatorname{Bd}(\tau)
$$

implies the inequality

$$
\tau^{\prime} \leqslant \gamma\left[\tau, \tau^{*}\right] .
$$

In particular, the mixed topology is the finest of all linear topologies which satisfy $\left(\mathrm{P}_{1}\right)$.

The proofs of lemmas 2.2.1. and 2.2.2. were given in my previous paper [12]. 
The topology $\tau^{\prime}$ is said to satisfy condition $\left(\mathrm{P}_{2}\right)$ (with respect to the pair $\left.\left(\tau, \tau^{*}\right)\right)$ provided

$\left(\mathbf{P}_{2}\right) \quad$ If $y=u(x)$ is a distributive operation defined on $X$ with values belonging to another topological linear space $\left\langle Y, \tau_{1}\right\rangle$, and if for each $Z \in \mathrm{Bd}(\tau)$ the operation $u \mid Z$ is $\left(\tau^{*} \mid Z, \tau_{1}\right)$-continuous, then the operation $u$ is $\left(\tau^{\prime}, \tau_{1}\right)$-linear.

2.2.3. If the topology $\tau$ satisfies oondition (o), then the topology $\gamma\left[\tau, \tau^{*}\right]$ satisfies the oondition $\left(\mathrm{P}_{2}\right)$.

2.2.4. Corollarx. If the topology $\tau$ satisfies condition (0), then a distributive operation $y=u(x)$ defined on $X$ with values belonging to another topological linear space $\left\langle Y, \tau_{1}\right\rangle$ is $\left(\tau^{\gamma}, \tau_{1}\right)$-continuous if and only if the operation $u \mid Z$ is $\left(\tau^{*} \mid Z, \tau_{1}\right)$-continuous for each $Z \in \mathrm{Bd}(\tau)$.

2.2.5. If the topology $\tau$ satisfies condition (o) and a linear topology $\tau^{\prime}$ satisfies condition $\left(\mathrm{P}_{2}\right)$, then $\tau^{\prime} \geqslant \tau^{\gamma}$. In other words, the topology $\tau^{\gamma}$ is the coarsest of all the linear topologies which satisfy $\left(\mathrm{P}_{2}\right)$.

The proofs of 2.2.3-2.2.5 were given in [1.2]. The following theorem is an immediate consequence of 2.2 .2 and 2.2 .5 :

2.2.6. THEOREM. If the topology $\tau$ satisfies condition (o) and if a linear topology $\tau^{\prime}$ satisfies conditions $\left(\mathrm{P}_{1}\right)$ and $\left(\mathrm{P}_{2}\right)$ simultaneously, then $\tau^{\prime}=\tau^{\gamma}$.

Henceforth we shall assume that the topology $\tau$ is locally convex and that $\mathfrak{U}(\tau)$ is a basis of convex neighbourhoods of 0 .

2.3. For each sequence $U_{n}^{*} \in \mathfrak{U}\left(\tau^{*}\right)(n=0,1,2, \ldots)$ and for each $U \in \mathfrak{U}(\tau)$ let us write

$$
U^{\nu_{1}}=\gamma_{1}\left(U_{0}^{*}, U_{1}^{*}, \ldots ; U\right)=U_{0}^{*} \cap \bigcap_{n=1}^{\infty}\left(n U+U_{n}^{*}\right) .
$$

We shall prove that the class of all the sets (6) is a basis of neighbourhoods for 0 in the mixed topology $\gamma\left[\tau, \tau^{*}\right]$. First we shall show, however, that if $\left\{a_{n}\right\}$ is an arbitrary sequence of positive numbers tending to infinity, then every set of the form

$$
V_{0}^{*} \cap \bigcap_{n=1}^{\infty}\left(a_{n} V+V_{n}^{*}\right), \quad \text { where } \quad V_{\in} \mathfrak{U}(\tau), V_{n}^{*} \in \mathfrak{U}\left(\tau^{*}\right),
$$

contains a set of form (6), and conversely. In fact, suppose that $U^{\gamma_{1}}$ is an arbitrary set of form (6). Let $\left\{k_{n}\right\}$ be an increasing sequence of positive integers such that $k_{n} \geqslant a_{n}$ for $n=1,2, \ldots$ Let $V_{n}^{*} \in \mathfrak{U}\left(\tau^{*}\right)$ be such a sequence that $\nabla_{0}^{*} \subset \bigcap_{p=0}^{k_{1}-1} U_{p}^{*}, \nabla_{n}^{*} \subset \bigcap_{p=k_{n}}^{k_{n+1}-1} U_{p}^{*}$. Then

$$
V_{0}^{*} \frown\left(a_{n} U+\nabla_{n}^{*}\right) \subset U_{0}^{*} \frown \bigcap_{p=k_{n}}^{k_{n+1}-1}\left(p U+U_{p}^{*}\right)
$$

and

$$
\nabla_{0}^{*} \frown \bigcap_{n=1}^{\infty}\left(\alpha_{n} U+\nabla_{n}^{*}\right) \subset U_{0}^{*} \frown \bigcap_{p=1}^{\infty}\left(p U+U_{p}^{*}\right) .
$$

It can be shown by a similar argument that, for every set of form (7), there are neighbourhoods $D^{*}{ }_{\epsilon} \mathfrak{U}\left(\tau^{*}\right), n=0,1,2, \ldots$, such that set $(7)$ contains set (6) for $U=\nabla$.

Now we shall show that every set (7) is a neighbourhood of 0 in the mixed topology. On account of the preceding remark it suffices to show that every set

$$
U_{0}^{*} \cap \bigcap_{n=1}^{\infty}\left(\frac{1}{2} n(n+1) U+U_{n}^{*}\right)
$$

is a neighbourhood of 0 in the mixed topology.

Let $\nabla_{1}^{*}$ be an arbitrary member of $\mathfrak{U}\left(\tau^{*}\right)$, satisfying the condition $\nabla_{1}^{*}+\nabla_{1}^{*} \subset U_{0}^{*}$. Let us take, by induction, $\nabla_{n}^{*} \in \mathfrak{U}\left(\tau^{*}\right)(n>1)$ such that

$$
\nabla_{n}^{*}+\nabla_{n}^{*} \subset U_{n-1}^{*} \frown \nabla_{n-1}^{*} \text {. }
$$

We have

$$
\nabla_{1}^{*}+\nabla_{2}^{*}+\ldots+\nabla_{n}^{*} \subset U_{0}^{*}
$$

and for every $p$

$$
\nabla_{n}^{*}+\nabla_{n+1}^{*}+\ldots+\nabla_{n+p}^{*} \subset \nabla_{n}^{*}+\nabla_{n}^{*} \subset \nabla_{n-1}^{*} .
$$

By (10), we obtain

$$
\begin{array}{r}
\gamma\left(\nabla_{1}^{*}, V_{2}^{*}, \ldots ; U\right)=\bigcup_{p=1}^{\infty}\left(\nabla_{1}^{*} \cap U+\nabla_{2}^{*} \frown 2 U+\ldots+\nabla_{n-1}^{*} \cap(n-1) U+\right. \\
\left.+V_{n}^{*} \frown n U+\ldots+\nabla_{n+p}^{*} \frown(n+p) U\right) \subset \bigcup_{p=1}^{\infty}(U+2 U+\ldots+(n-1) U+ \\
\left.+V_{n}^{*}+\ldots+\nabla_{n+p}^{*}\right) \subset \frac{1}{2} n(n-1) U+U_{n-1}^{*} .
\end{array}
$$

The last inclusion being valid for each $n>1$, it follows from (9) that set (8) contains the set $\gamma\left(\nabla_{1}^{*}, \nabla_{2}^{*}, \ldots ; U\right)$. Therefore set $(8)$ is a neighbourhood of 0 in the mixed topology.

Now we shall show that every neighbourhood of 0 in the mixed topology contains a set of form (6). Let $U^{\gamma}=\gamma\left(U_{1}^{*}, Z_{2}^{*}, \ldots ; U\right)$ be a neighbourhood of 0 in the mixed topology. Let us write, for brevity, $m_{n}=2 n-1 \quad(n=1,2, \ldots)$. There exists a sequence $\nabla_{0}^{*}, \nabla_{1}^{*}, \ldots$, such that $\nabla_{0}^{*}+\nabla_{0}^{*} \subset U_{m_{1}}^{*}, \nabla_{p-1}^{*}+\nabla_{p-1}^{*} \subset D_{m_{p}}^{*}, \nabla_{p-1}^{*} \supset \nabla_{p}^{*}(p=1,2, \ldots)$. We shall prove that $\nabla^{\gamma_{1}}=\gamma_{1}\left(\nabla_{0}^{*}, \nabla_{1}^{*}, \ldots ; U\right) \subset U^{\gamma}$. Let $x \in \nabla^{\gamma_{1}}$. Then $x \in V_{0}^{*}$ and, for each $n=1,2, \ldots$, there exists a decomposition $x=y_{n}+z_{n}$ 
where $y_{n} \epsilon n U, z_{n} \epsilon V_{n}^{*}$. Let $x_{1}=y_{1}$ and $x_{n}=y_{n}-y_{n-1}$ for $n>1$. We have, for every $n$, the following obvious identity:

$$
\begin{gathered}
x_{1}+x_{2}+\ldots+x_{n}+z_{n}=y_{1}+\left(y_{2}-y_{1}\right)+\ldots+ \\
+\left(y_{n}-y_{n-1}\right)+z_{n}=y_{n}+z_{n}=x .
\end{gathered}
$$

Furthermore

$$
z_{n-1}=x_{n}+z_{n},
$$

and therefore $x_{n}=z_{n-1}-z_{n} \in V_{n-1}^{*}+V_{n}^{*}$. On the other hand, $x_{n}=y_{n}-y_{n-1}$ $\epsilon n U+(n-1) U=(2 n-1) U=m_{n} U$. Hence

$$
x_{n} \epsilon\left(V_{n-1}^{*}+V_{n}^{*}\right) \frown(2 n-1) U .
$$

It follows immediately from the definition of $V_{n}^{*}$ that

$$
V_{n}^{*}+V_{n}^{*} \subset V_{n-1}^{*}+V_{n-1}^{*} \subset U_{m_{n}}^{*}
$$

Hence

$$
x_{n} \in U_{m_{n}}^{*} \frown m_{n} U \text {. }
$$

It follows from the equality $z_{n}=x-y_{n}$ that $z_{n} \epsilon\left(k_{0}+n\right) U$ where $k_{0}$ is a positive number such that $x \in k_{0} U$. If $n_{0}>k_{0}-1$, then $2 n_{0}+1=m_{n_{0+1}}$ $>k_{0}+n_{0}$ and $z_{n_{0}} \epsilon\left(k_{0}+n_{0}\right) U \subset m_{n_{0}+1} U$. On the other hand,

$$
z_{n_{0}} \epsilon V_{n_{0}}^{*} \subset V_{n_{0}}^{*}+V_{n_{0}}^{*} \subset U_{m_{n_{0}+1}}^{*}
$$

Therefore

$$
z_{n_{0}} \in U_{m_{0+1}}^{*} \cap m_{n_{0}+1} U .
$$

It follows from (11), (12) and (13) that

$$
\begin{aligned}
x=x_{1}+x_{2}+\ldots+x_{n_{0}}+z_{n_{0}} \epsilon U_{m_{1}}^{*} \cap m_{1} U+\ldots+ \\
+\quad U_{m_{n_{0}}}^{*} \cap m_{n_{0}} U+U_{m_{n_{0}+1}}^{*} \cap m_{n_{0}+1} U \subset U^{\eta^{\prime}} .
\end{aligned}
$$

This proves that $V^{\gamma_{1}} \subset U^{\nu}$.

Therefore all sets (6) (or all sets (7)) form a basis of neighbourhoods of 0 in the mixed topology.

If, in particular, $\tau$ is a normed topology defined by the norm \|\| and $S_{n}=[x:\|x\| \leqslant n]$, then the sets

$$
U_{0}^{*} \cap \bigcap_{n=1}^{\infty}\left(r S_{n}+U_{n}^{*}\right), \quad U_{n}^{*} \in \mathfrak{U}\left(\tau^{*}\right), \quad r>0,
$$

compose a basis of neighbourhoods of 0 in the mixed topology.
Remark. If the topologies $\tau$ and $\tau^{*}$ are locally convex and if condition (o) is satisfied, then the class of all the sets

$$
\operatorname{conv} \bigcup_{n=1}^{\infty}\left(U_{n}^{*} \cap n U\right)
$$

is also a basis of neighbourhoods of 0 in the mixed topology.

In fact, it is clear that sets (15) form a basis of neighbourhoods for a locally convex linear topology $\tau_{1}$. The inequality $\tau_{1} \geqslant \tau^{\gamma}$ is obvious. The inverse inequality follows from 2.2.2, because the topology $\tau_{1}$ has the property $\left(\mathrm{P}_{1}\right)$.

2.3.1. THEOREM. Suppose that the topologies $\tau$ and $\tau^{*}$ satisfy condition (d). Then $x_{n} \rightarrow x_{0}$ in the mixed topology if and only if simultaneously

a) $x_{n} \rightarrow x_{0}$ in the $\tau^{*}$-topology,

b) the sequence $\left\{x_{n}\right\}$ is bounded in the $\tau$-topology.

Proof $\left({ }^{3}\right)$. Let us suppose that $x_{n} \rightarrow x_{0}$ in the $\tau^{*}$-topology and the sequence $\left\{x_{n}\right\}$ is $\tau$-bounded. Let $Z$ be set of all elements $x_{n}, n=0,1, \ldots$ We have

$$
x_{n} \rightarrow x_{0} \quad \text { in } \quad \tau^{*} \mid Z \text {-topology } .
$$

The $\tau$-boundedness of $Z$ implies the equality $\tau^{*}\left|Z=\tau^{\nu}\right| Z$, on account of the proposition 2.2.1. Hence $x_{n} \rightarrow x_{0}$ in $\tau^{\gamma} \mid Z$-topology, i. e. $x_{n} \rightarrow x_{0}$ in the mixed topology $\tau^{\gamma}$.

Suppose now that $x_{n} \rightarrow x_{0}$ in the mixed topology. It follows from the inequality $\tau^{*} \leqslant \tau^{\gamma}$ that $x_{n} \rightarrow x_{0}$ in the $\tau^{*}$-topology. It suffices to prove that $\left\{x_{n}\right\}$ is $\tau$-bounded. Suppose the contrary. We may assume that $x_{0}=0$, i. e. that $x_{n} \rightarrow 0$ in the mixed topology. If the sequence $\left\{x_{n}\right\}$ is not $\tau$-bounded, then there exists a neighbourhood $U \in \mathfrak{U}(\tau)$ and an increasing sequence of indices $\left\{k_{n}\right\}$ such that $x_{k_{n}} \notin n U$ for $n=1,2, \ldots$ It follows from condition (d) that all the sets $n U$ are $\tau^{*}$-closed. Hence, for each $n$, there exists $U_{n}^{*} \in \mathfrak{U}\left(\tau^{*}\right)$ such that $x_{k_{n}} \notin n U+{U_{n}^{*}}_{n}$. Therefore the set $U^{\gamma_{1}}=\bigcap_{n=1}^{\infty}\left(n U+U_{n}^{*}\right)$ contains no of the elements $x_{k_{n}}$. On the other hand, the set $U^{\gamma_{1}}$ is a neighbourhood of 0 in the mixed topology. This contradicts the hypothesis that $\left\{x_{n}\right\}$ converges to 0 in the mixed topology. Therefore the sequence $\left\{x_{n}\right\}$ is $\tau$-bounded.

2.3.2. CoRollary. Under the hypotheses of theorem 2.3.1, a sequence $\left\{x_{n}\right\}$ is a Cauchy sequence in the mixed topology if and only if simultaneously
a) $\left\{x_{n}\right\}$ is a Cauchy sequence in the $\tau^{*}$-topology

b) $\left\{x_{n}\right\}$ is r-bounded.

$\left({ }^{3}\right)$ I have proved this theorem in paper [12]. The proof given here is simpler than that in [12]. 
2.4. We suppose in this section that the topologies $\tau$ and $\tau^{*}$ satisfy conditions (o) and (d).

2.4.1. A set $Z \subset X$ is bounded in the mixed topology if and only if it is bounded in topologies $\tau$ and $\tau^{*}$ simultaneously. In symbols

$$
\operatorname{Bd}\left(\tau^{\gamma}\right)=\mathrm{Bd}(\tau) \frown \mathrm{Bd}\left(\tau^{*}\right) .
$$

Proof. Suppose that $A \in \mathrm{Bd}(\tau) \frown \mathrm{Bd}\left(\tau^{*}\right)$. If $x_{n} \in A$ and $\lambda_{n} \geqslant 0, \lambda_{n} \rightarrow 0$, then $\lambda_{n} x_{n} \rightarrow 0$ in both topologies $\tau$ and $\tau^{*}$. Hence the sequence $\left\{\lambda_{n} x_{n}\right\}$ is $\tau$-bounded. In virtue of theorem 2.3.1 we infer that $\lambda_{n} x_{n} \rightarrow 0$ in the mixed topology, and consequently $A \in \operatorname{Bd}\left(\tau^{\gamma}\right)$. Therefore $\operatorname{Bd}(\tau) \cap \operatorname{Bd}\left(\tau^{*}\right)$ $C \operatorname{Bd}\left(\tau^{\gamma}\right)$. Inequality (2) implies the inclusion $B d\left(\tau^{\nu}\right) \subset B d\left(\tau^{*}\right)$. Suppose that $A \in \operatorname{Bd}\left(\tau^{\nu}\right)$. Let $\left\{x_{n}\right\}$ be a sequence of elements of the set $A$, and let $\lambda_{n} \geqslant 0, \lambda_{n} \rightarrow 0$. Since $\sqrt{\lambda_{n}} \rightarrow 0$, we have $\sqrt{\lambda_{n}} x_{n} \rightarrow 0$ in the mixed topology. In virtue of theorem 2.3.1 the sequence $\left\{\sqrt{\lambda_{n}} x_{n}\right\}$ is $\tau$-bounded. Consequently $\sqrt{\lambda_{n}} \cdot \sqrt{\lambda_{n}} x_{n}=\lambda_{n} x_{n} \rightarrow 0$ in the $\tau$-topology and therefore the set $A$ is $\tau$-bounded. Hence $\mathrm{Bd}\left(\tau^{\gamma}\right) \subset \mathrm{Bd}(\tau)$.

CoROLlarx. If the topologies $\tau$ and $\tau^{*}$ satisfy condition (n), then a set $A \subset X$ is bounded in the mixed topology if and only if it is $\tau$-bounded.

2.4.2. If $\gamma\left[\tau, \tau^{*}\right]=\tau$, then $\tau^{*} \geqslant \tau$. If, in particular, $\tau^{*} \leqslant \tau$, then the equality $\gamma\left[\tau, \tau^{*}\right]=\tau$ implies $\tau=\tau^{*}$.

Proof. Let $V$ be any $\tau$-bounded neighbourhood of 0 in the $\tau$-topology. By hypothesis, $V$ contains a neighbourhood $\gamma\left(U_{1}^{*}, U_{2}^{*}, \ldots, ; U\right)$. It follows from the $\tau$-boundedness of the set $V$, that $V \subset n_{0} U$ for some $n_{0} \geqslant 1$. We shall show that $U_{n_{0}+1}^{*} \subset V$. Suppose this inclusion is false Then there exists $x_{0} \in U_{n_{0}+1}^{*}, x_{0} \oplus V$. It is elear that $\lambda x_{0} \notin V, \lambda x_{0} \in\left(n_{0}+1\right) U$ for a number $\lambda, 0<\lambda \leqslant 1$. We have $\lambda x_{0} \in U_{n_{0}+1}^{*}$ (see condition $\left(1_{2}\right)$ ). Hence

$$
\lambda x_{0} \in D_{n_{0+1}}^{*} \cap\left(n_{0}+1\right) U \subset \gamma\left(\sigma_{1}^{*}, U_{2}^{*}, \ldots ; U\right) \subset V .
$$

This contradicts the assumption ' $\lambda x_{0} \notin V$. Therefore $U_{n_{0}+1}^{*} \subset V$, and consequently $\tau^{*} \geqslant \tau$.

$$
\text { 2.4.3. If } \tau_{1}^{*}\left|Z=\tau_{2}^{*}\right| Z \text { for each } Z \in \mathrm{Bd}(\tau) \text {, then }
$$

$$
\text { In particular: }
$$

$$
\gamma\left[\tau, \tau_{1}^{*}\right]=\gamma\left[\tau, \tau_{2}^{*}\right] .
$$

$$
\gamma\left[\tau, \tau^{*}\right]=\gamma\left[\tau, \gamma\left[\tau, \tau^{*}\right]\right]
$$

Proof. By 2.2.1,

$$
\gamma\left[\tau, \tau_{2}^{*}\right]\left|Z=\tau_{2}^{*}\right| Z=\tau_{1}^{*} \mid Z \quad \text { for } \quad Z \in \operatorname{Bd}(\tau) .
$$

Therefore the topology $\gamma\left[\tau, \tau_{2}^{*}\right]$ has property $\left(\mathrm{P}_{1}\right)$ (for $\tau^{*}=\tau_{1}^{*}$ ). Consequently $\gamma\left[\tau, \tau_{1}^{*}\right] \geqslant \gamma\left[\tau, \tau_{2}^{*}\right]$, by 2.2.2. Replacing $\tau_{1}^{*}$ by $\tau_{2}^{*}$ and conversely we obtain the inverse inequality. Hence equality (16) is true. Setting in (16) $\tau_{1}^{*}=\tau^{*}, \tau_{2}^{*}=\gamma\left[\tau, \tau^{*}\right]$ we obtain equality (17).

It follows from 2.4.3 that, contrary to the case considered in 2.4.2, the equality $\gamma\left[\tau, \tau^{*}\right]=\tau^{*}$ does not imply the equality $\tau=\tau^{*}$. In fact, if $\tau_{1}^{*} \leqslant \tau, \tau_{1}^{*} \neq \tau$ and $\tau^{*}=\gamma\left[\tau, \tau_{1}^{*}\right]$, then $\tau^{*} \leqslant \tau, \quad \tau^{*} \neq \tau$ and $\tau^{*}$ $=\gamma\left[\tau, \tau^{*}\right]$.

2.4.4. If condition (n) is satisfied and if $\left\langle X, \gamma\left[\tau, \tau^{*}\right]\right\rangle$ is a bornological space, then $\tau=\tau^{*}$.

Proof. By 2.4.1 (corollary) we have $\mathrm{Bd}\left(\gamma\left[\tau, \tau^{*}\right]\right)=\mathrm{Bd}(\tau)$. Therefore $\gamma\left[\tau, \tau^{*}\right] \geqslant \tau$, by $(*)$. Hence $\gamma\left[\tau, \tau^{*}\right]=\tau$, on account of inequality (3). This equality implies $\tau=\tau^{*}$, by 2.4 .2 .

2.4.5. If condition (n) is satisfied and if $\left\langle X, \gamma\left[\tau, \tau^{*}\right]\right\rangle$ is a t-space, then $\tau=\tau^{*}$.

Proof. Let $U \in \mathfrak{U}(\tau)$. By condition (d), the neighbourhood $U$ is $\tau^{*}$-closed. It follows from the inequality $\tau^{*} \leqslant \gamma\left[\tau, \tau^{*}\right]$ that $U$ is closed in the mixed topology. Therefore the set $U$ is a barrel in the space $\left\langle X, \gamma\left[\tau, \tau^{*}\right]\right\rangle$ and consequently $U$ is a neighbourhood of 0 in the mixed topology. Hence $\gamma\left[\tau, \tau^{*}\right] \geqslant \tau$, and, by 2.4.2, $\tau=\tau^{*}$.

Theorems 2.4.4 and 2.4.5 show that, in non-trivial cases, spaces with mixed topology fail to be bornological or tonnelé.

2.5. Let $X_{0}$ be a linear subspace of the space $X$. We shall consider on the space $X_{0}$ the following two topologies:

a) the topology $\gamma\left[\tau, \tau^{*}\right] \mid X_{0}$, i. e. the topology induced on $X_{0}$ by the mixed topology $\gamma\left[\tau, \tau^{*}\right]$.

b) the topology $\gamma\left[\tau\left|X_{0}, \tau^{*}\right| X_{0}\right]$, i. e. the mixed topology constructed from the topologies induced on $X_{0}$ by $\tau$ and $\tau^{*}$.

It is easy to verify that

In fact, the sets

$$
\gamma\left[\tau, \tau^{*}\right] \mid X_{0} \leqslant \gamma\left[\tau\left|X_{0}, \tau^{*}\right| X_{0}\right]
$$

$$
X_{0} \cap{J_{0}^{*}}_{n} \cap \bigcap_{n=1}^{\infty}\left(U_{n}^{*}+n U\right)
$$

are neighbourhoods of 0 in the topology $\gamma\left[\tau, \tau^{*}\right] \mid X_{0}$, and the sets

$$
X_{0} \frown U_{0}^{*} \frown \bigcap_{n=1}^{\infty}\left(D_{n}^{*} \cap X_{0}+n U \frown X_{0}\right)
$$

are neighbourhoods of 0 in the topology $\gamma\left[\tau\left|X_{0}, \tau^{*}\right| X_{0}\right]$. It is clear that the second set is contained in the first. The inverse inclusion is, in general, false.

Suppose now that the subspace $X_{0}$ and the topologies $\tau$ and $\tau^{*}$ satisfy the following condition: 
$\left(c_{1}\right)$ if $A \subset X, A \in \mathrm{Bd}(\tau), A$ is $\tau^{*}$-closed and $A \cap X_{0}=\varnothing$, then there exists $V^{*} \in \mathfrak{U}\left(\tau^{*}\right)$ such that $\left(A+V^{*}\right) \frown X_{0}=\varnothing$.

Condition $\left(c_{1}\right)$ is satisfied, in particular, if $X_{0}$ is $\tau^{*}$-closed and if every $\tau$-bounded $\tau^{*}$-closed set is compact (= bicompact) in the $\tau^{*}$-topology.

2.5.1. THEOREM. Suppose that conditions (d) and (o) are satisfied. Then condition $\left(\mathrm{c}_{1}\right)$ implies the equality

$$
\gamma\left[\tau\left|X_{0}, \tau^{* *}\right| X_{0}\right]=\gamma\left[\tau, \tau^{*}\right] \mid X_{0} .
$$

Proof. Let $U_{0}^{*} \frown \bigcap_{n=1}^{\infty}\left(U_{n}^{*} \cap X_{0}+n U \cap X_{0}\right)$ be a neighbourhood of 0 in the topology $\gamma\left[\tau\left|X_{0}, \tau^{*}\right| X_{0}\right]$. On account of conditions (d) and (o) we may assume that the sets $n U$ are $\tau^{*}$-closed and $\tau$-bounded. Let $W^{*}$ be a $\tau^{*}$-open $\tau^{*}$-neighbourhood of 0 , such that $W_{n}^{*}+W_{n}^{*} \subset U_{n}^{*}$. The set $n U \frown X_{0}+W_{n}^{*}$ is $\tau^{*}$-open. Hence the set

$$
A_{n}=n U \backslash\left(n U \frown X_{0}+W_{n}^{* *}\right)
$$

is $\tau^{*}$-closed. Since the set $A_{n}$ is $\tau$-bounded and $A_{n} \cap X_{0}=\varnothing$, then, by condition $\left(\mathbf{c}_{1}\right)$, there exists $V_{n}^{*}{ }_{\epsilon} \mathcal{L}\left(\tau^{*}\right)$ such that $\left(A_{n}+V_{n}^{*}\right) \frown X_{0}=\varnothing$. We may suppose that $V_{n}^{*} \subset W_{n}^{*}$ and therefore $W_{n}^{*}+V_{n}^{*} \subset U_{n}^{*}$.

We have

$$
\begin{aligned}
& X_{0} \cap\left(n U+V_{n}^{*}\right) \subset X_{0} \frown\left[\left(A_{n}+V_{n}^{*}\right) \cup\left(n U \cap X_{0}+W_{n}^{*}+V_{n}^{*}\right)\right] \\
& \subset X_{0} \frown\left[\left(A_{n}+V_{n}^{*}\right) \cup\left(n U \cap X_{0}+U_{n}^{*}\right)\right]=X_{0} \frown\left(n U \cap X_{0}+U_{n}^{*}\right) \\
& \quad=n U \frown X_{0}+U_{n}^{*} \cap X_{0} .
\end{aligned}
$$

The last equality follows from the fact that the set $X_{0}$ is linear. Therefore

$$
X_{0} \frown U_{0}^{*} \frown \bigcap_{n=1}^{\infty}\left(n U+V_{n}^{*}\right) \subset X_{0} \frown U_{0}^{*} \frown \bigcap_{n=1}^{\infty}\left(n U \frown X_{0}+U_{n}^{*} \frown X_{0}\right),
$$

which implies $\gamma\left[\tau, \tau^{*}\right] \mid X_{0} \geqslant \gamma\left[\tau\left|X_{0}, \tau^{*}\right| X_{0}\right]$. The inverse inequality being always valid, we get equality (18).

2.6. Let $X$ be a linear space with a homogeneous norm \|| I, and let \|\|$^{*}$ be another $F$-norm defined on the space $X$. A sequence $\left\{x_{n}\right\}$ of elements of the space $X$ is said to be $\gamma$-convergent to $x_{0}$, in symbols $x_{n,} \rightarrow x_{0}$, if $\left\|x_{n}-x_{0}\right\|^{*} \rightarrow 0$ and $\sup \left\|x_{n}\right\|<\infty$. The $\gamma$-convergence is also called two-norm convergence. The space $X$ with $\gamma$-convergence is denoted by $\left\langle X,\|\|,\|\|^{*}\right\rangle$ and called a two-norm space. The theory of two-norm spaces has been developed by Alexiewicz ([1], [2]). Two-norm convergence in some concrete spaces was examined earlier by G. Fichtenholz [7]. The following conditions are important in the theory of two-norm spaces:
$\left(\mathrm{n}_{1}\right) \quad\left\|x_{n}\right\| \rightarrow 0$ implies $\left\|x_{n}\right\|^{*} \rightarrow 0$,
$\left(\mathrm{n}_{2}\right) \quad\left\|x_{n}-x_{0}\right\|^{*} \rightarrow 0$ implies liminf $\left\|x_{n}\right\| \geqslant\left\|x_{0}\right\|$,

$\left(\mathrm{n}_{3}\right) \quad$ if $\left\|x_{n}\right\| \leqslant K, \lim _{p \rightarrow \infty}\left\|x_{p}-x_{q}\right\|^{*}=0$, then there exists $x_{0} \in X$ such $\lim _{\substack{p \rightarrow \infty \\ q \rightarrow \infty}}$

that $\left\|x_{0}\right\| \leqslant K$ and $\left\|x_{n}-x_{0}\right\|^{*} \rightarrow 0$.

Let $\tau$ be the linear topology defined by the norm \|\| , and let $\tau^{*}$ be the linear topology defined by the norm \|\|$^{*}$. Let $\mathfrak{U}(\tau)$ be the class of all solid spheres $S_{r}=[x:\|x\| \leqslant v]$. Condition (0) from 2.1. is obviously satisfied. Condition (n) from 2.1 is identical with $\left(\mathrm{n}_{1}\right)$, and condition (d) is identical with $\left(\mathrm{n}_{2}\right)$.

If $\left\langle Y, \tau_{1}\right\rangle$ is a topological linear space and if $y=u(x)$ is a distributive operation defined on $X$ with values in $Y$, then the operation $u$ is said to be $\left(\gamma, \tau_{1}\right)$-linear provided $x_{n} \rightarrow x_{0}$ implies $u\left(x_{n}\right) \underset{\tau_{1}}{ } u\left(x_{0}\right)$. In particular, a distributive functional $\xi(x)$ defined on $X$ is said to be $\gamma$-linear provided $x_{n} \rightarrow x_{0}$ implies $\xi\left(x_{n}\right) \rightarrow \xi\left(x_{0}\right)$.

The connection between the two-norm spaces and the spaces with mixed topology is stated by the following theorem:

2.6.1. THEOREM. A) If $\left\langle\bar{X},\|\|,\|\|^{*}\right\rangle$ is a two-norm space satisfying condition $\left(\mathrm{n}_{2}\right)$, then the mixed topology $\tau^{\gamma}=\gamma\left[\tau, \tau^{*}\right]$ has the following properties:

(i) $\tau^{\gamma}\left|S=\tau^{*}\right| S$, where $S=[x:\|x\| \leqslant 1]$;

(ii) $x_{n} \rightarrow x_{0}$ if and only if $x_{n} \rightarrow x_{0}$ in the $\tau^{\gamma}$-topology;

(iii) for every linear topological space $\left\langle Y, \tau_{1}\right\rangle$, and for every operation $y=u(x)$ from $X$ to $Y, u$ is $\left(\gamma, \tau_{1}\right)$-linear if and only if it is $\left(\tau^{\gamma}, \tau_{1}\right)$-linear.

B) The mixed topology $\gamma\left[\tau, \tau^{*}\right]$ is a unique linear topology possessing properties (i) and (iii).

Proof of A). Property (i) follows from 2.2.1. Property (ii) is an immediate consequence of theorem 2.3.1. A distributive operation $u$ is $\left(\gamma, \tau_{1}\right)$-linear if and only if the operation $u \mid S$ is $\left(\tau^{*} \mid S, \tau_{1}\right)$-continuous, or, which is the same, if and only if, for each $Z \in \operatorname{Bd}(\tau)$ the operation $u \mid Z$ is $\left(\tau^{*} \mid Z, \tau_{1}\right)$-continuous. Therefore property (iii) follows at once from 2.2.4.

Proof of B). Suppose that a topology $\tau^{\prime}$ has properties (i) and (iii). It follows from (i) that the topology $\tau^{\prime}$ satisfies condition $\left(P_{1}\right)$ from 2.2. Hence $\tau^{\prime} \leqslant \tau^{\gamma}$, by 2.2.2. If the operation $u$ from $X$ to $Y$ is distributive and $u \mid S^{\prime}$ is $\left(\tau^{*} \mid S, \tau_{1}\right)$-continuous, then, by condition (iii), $u$ is $\left(\tau^{\prime}, \tau_{1}\right)$ linear. Consequently, the topology $\tau^{\prime}$ satisfies condition $\left(\mathrm{P}_{2}\right)$ from 2.2 , and $\tau^{\prime} \geqslant \tau^{\gamma}$ by 2.2.5. Consequently $\tau^{\prime}=\tau^{\gamma}$.

There exists a close relation between two-norm spaces and Saks spaces. The theory of Saks spaces has been developed by W. Orlicz ([9], [10]). His definitions are as follows. Let $X_{s}=S$ be the unit solid sphere in a normed space $\langle X,\|\|\rangle$, and let \|\|$^{*}$ be another $F$-norm defined on $X$. If the set $X_{s}$ with the metric $d\left(x_{1}, x_{2}\right)=\left\|x_{1}-x_{2}\right\|^{*}\left(x_{1}, x_{2} \in X_{s}\right)$ is a com- 
plete metric space (i. e. if condition $\left(\mathrm{n}_{3}\right)$ is satisfied), then the space $\left\langle X_{s}, \tau^{*} \mid X_{s}\right\rangle$ is called a Saks space. Let $\left\langle Y, \tau_{\mathbf{1}}\right\rangle$ be any topological linear space and let $y=u(x)$ be an operation defined on the space $X_{s}$ with values in $Y$. The operation $u$ is called distributive if the conditions $\lambda_{1}, \lambda_{2} \epsilon \mathcal{R}$, $x_{1}, x_{2} \in X_{s}, \lambda_{1} x_{1}+\lambda_{2} x_{2} \in X_{s}$ imply $u\left(\lambda_{1} x_{1}+\lambda_{2} x_{2}\right)=\lambda_{1} u\left(x_{1}\right)+\lambda_{2} u\left(x_{2}\right)$. If the operation $u$ is distributive and $\left(\tau^{*} \mid X_{s}, \tau_{1}\right)$-continuous, then $u$ is called $\left(X_{s}, Y\right)$-linear.

As long as we deal with convergence and linear operations, it makes no difference whether we consider Saks spaces or two-norm spaces. In fact, if $x_{n} \rightarrow x_{0}$ in the Saks space $\left\langle X_{s}, \tau^{*} \mid X_{s}\right\rangle$, then $x_{n} \rightarrow x_{0}$ in the twonorm space $\left\langle X,\|\|,\|\|^{*}\right\rangle$. Conversely, if $x_{n} \rightarrow x_{0}$ in the space $\left\langle X,\|\|,\|\|^{*}\right\rangle$, then there exists $\lambda \epsilon \Re, \lambda \neq 0$ such that $\lambda x_{n} \in X_{s}, \lambda x_{0} \in X_{s}$ and $\lambda x_{n} \rightarrow \lambda x_{0}$ in the space $\left\langle X_{s}, \tau^{*} \mid X_{s}\right\rangle$. If $y=u(x)$ is a $\left(X_{s}, Y\right)$-linear operation from $X_{s}$ to $\left\langle Y, \tau_{1}\right\rangle$, then $u$ may be extended in a unique manner to a $\left(\gamma, \tau_{1}\right)$ linear operation defined on the whole space $X$. Conversely, if $u$ is a $\left(\gamma, \tau_{1}\right)$ linear operation from $X$ to $Y$, then the operation $u \mid X_{s}$ is $\left(X_{s}, Y\right)$-linear.

The following theorem states the relation between condition $\left(\mathrm{n}_{3}\right)$ and the properties of mixed topology:

2.6.2. Condition $\left(\mathrm{n}_{3}\right)$ is satisfied if and only if simultaneously

a) the topologies $\tau$ and $\tau^{*}$ satisfy condition (d) from 2.1,

b) the space $\left\langle X, \tau^{\gamma}\right\rangle$ is sequentially complete.

This theorem easily follows from theorem 2.3.1 and corollary 2.3.2.

It follows from a theorem due to D. A. Raikov ([11], p. 223) that 2.6.2. may be strengthened as follows:

2.6.3. If condition $\left(\mathrm{n}_{3}\right)$ is satisfied, then the space $\left\langle X, \tau^{\nu}\right\rangle$ is complete, i. e. every Cauchy filter on $\left\langle\dot{X}, \tau^{\gamma}\right\rangle$ converges to a point of the space.

It follows from theorem 2.6.1 that a distributive operation from $\left\langle X, \tau^{\gamma}\right\rangle$ into another topological linear space is continuous if and only if it is sequentially continuous. Thus we see that the space $\left\langle X, \tau^{\gamma}\right\rangle$, although it fails to be bornological (see 2.4.4), has in this case the following important property of bornological spaces: the notions of continuity and sequential continuity of operations defined on this space coincide. A. Alexiewicz and $\mathrm{Z}$. Semadeni have shown [3] that if $\tau^{*}$ is nonmetrizable, then the space $\left\langle X, \tau^{\gamma}\right\rangle$ does not always possess this property. The example due to Alexiewicz and Semadeni is as follows. Let $X$ be the space of all bounded measurable functions $x=x(t)$ defined on $\langle 0,1\rangle$. The $\tau$-topology is defined by the norm $\|x\|=\sup |x(t)|$, and the $\tau^{*}$-topology is defined by the set of pseudonorms $\|x\|_{t}^{*}=|x(t)|$

The functional ${ }_{1}(x)=\tau_{0}$ The functional $\xi(x)=\int_{0}^{1} x(t) d t$, defined on the space $\left\langle X, \tau^{\gamma}\right\rangle$, is sequentially continuous, but not continuous.
Alexiewicz and Semadeni [4] have constructed another linear topology generating $\gamma$-convergence. The procedure applied by Alexiewiez and Semadeni requires the hypothesis that the space $\left\langle X,\|\|^{*}\right\rangle$ is $B_{0}^{*}$ space. Their definition is as follows: Suppose that condition $\left(n_{1}\right)$ is satisfied. Let $E$ be the conjugate space to $\langle X,\|\|\rangle$ with the usual norm. $\|\xi\|=\sup _{\|x\| \leq 1}|\xi(x)|$. Let $\Xi_{\gamma}$ be the space of all $\gamma$-linear functionals defined on $\left\langle X,\|\|,\|\|^{*}\right\rangle$. Since $\Xi_{\gamma} \subset \Xi$, the norm $\|\xi\|$ is defined for each $\xi \in \Xi_{\gamma}$. Let $U^{*}{ }_{\epsilon} \mathfrak{U}\left(\tau^{*}\right), \xi_{n} \in \Xi_{\gamma},\left\|\xi_{n}\right\| \leqslant 1,0<\alpha_{n} \rightarrow \infty$. We write

$$
\nabla\left(U^{*},\left\{\xi_{n}\right\},\left\{a_{n}\right\}\right)=U^{*} \cap \bigcap_{n=1}^{\infty}\left[x:\left|\xi_{n}(x)\right| \leqslant a_{n}\right] .
$$

The sets $V\left(U^{*},\left\{\xi_{n}\right\},\left\{\alpha_{n}\right\}\right)$ constitute a basis of neighbourhoods for 0 in a locally convex linear topology on $X$. This is the topology $\mu$ of Alexiewicz and Semadeni. The topology $\mu$ has the following properties ([4], p. 127):

( $\alpha) x_{n} \rightarrow x_{0}$ if and only if $x_{n} \rightarrow x_{0}$,

( $\beta$ ) the $\gamma$-linear functionals are identical with the functionals linear with respect to the topology $\mu$.

Following Alexiewicz and Semadeni a linear locally convex Hausdorff topology $\tau^{\prime}$ is called appropriate if it satisfies conditions $(\alpha)$ and $(\beta)$, i. e. if for sequences $\gamma$-convergence is equivalent to $\tau^{\prime}$-convergence, and if the class of $\gamma$-linear functionals is identical with the class of functionals linear in the topology $\tau^{\prime}$. Alexiewicz and Semadeni have shown that there exist different appropriate topologies for the space $\left\langle X,\|\|,\|\cdot\|^{*}\right\rangle$ ([4], p. 134). It follows from theorem 2.6.1 that, if we modify the definition of "appropriate" topology taking conditions (i) and (iii) in the place of $(\alpha)$ and $(\beta)$, then the "appropriate" topology is determined uniquely.

Theorem 2.6.1 enables us to apply the mixed topology to the study of two-norm spaces. For example, the problem of extension of $\gamma$-linear functionals is closely connected with the problem of relativization of the mixed topology to a linear subspace. This is shown by the following theorem:

2.6.4. Theorem. Let $\left\langle X,\|\|,\|\|^{*}\right\rangle$ be a two-norm space such that $\left\langle X,\|\|^{*}\right\rangle$ is a $B_{0}^{*}$-space. Let $X_{0}$ be a linear subspace of the space $X$ such that

$$
\gamma\left[\tau, \tau^{*}\right] \mid X_{0}=\gamma\left[\tau\left|X_{0}, \tau^{*}\right| X_{0}\right]
$$

Let $\xi_{0}$ be some $\gamma$-linear functional on $X_{0}$. Then there exists a $\gamma$-linear extension $\xi$ of $\xi_{0}$ on the whole space $X$.

Theorem 2.6.4 is an immediate consequence of theorem 2.6.1 and 
of the well-known theorem on extension of linear functionals in locally convex spaces.

Alexiewicz and Semadeni [4] have shown that $\gamma$-linear functionals do not have, in general, the extension property. Honce, equality (18) is not true in general. However, we considered in 2.5 a case where equality (18) was true. From theorems 2.5.1. and 2.6.3 we obtain

2.6.5. Theorem. Let $\left\langle X,\|\|,\|\|^{*}\right\rangle$ be a two-norm space such that $\left\langle X,\|\|^{*}\right\rangle$ is a $B_{0}^{*}$-space and such that the following condition is satisfied:

(c) the sphere $S^{\prime}=[x:\|x\| \leqslant 1]$ is $\tau^{*}$-compact.

If a tinear subspace $X_{0} \subset X$ is $\tau^{*}$-closed and if $\xi_{0}$ is a $\gamma$-linear functional on $X_{0}$, then there exists a $\gamma$-linear extension $\xi$ of $\xi_{0}$ on the whole space $X$.

Another theorem on extension of $\gamma$-linear functionals for two-norm spaces which are simultaneously vector lattices, has been proved by Alexiewicz and Semadeni [3].

3. We shall now give some examples of spaces with mixed topology. These examples will be preceded by theorem 3.1.1 which enables us to establish the form of $\tau^{\gamma}$-neighbourhoods in many concrete spaces.

3.1. Suppose that in a linear space $X$ the topology $\tau$ is defined by a homogeneous norm \|\| , and the topology $\tau^{*}$ is defined by a set (uncountable, in general) of homogeneous pseudonorms \|\|$_{\beta}^{*}(\beta \in B)$. Suppose, moreover, that the norm \|\| and the psendonorms \|\|$_{\beta}^{*}$ satisfy the condition

$$
\|x\|=\sup _{\beta \in B}\|x\|_{\beta}^{*} \quad \text { for each } \quad x \in X .
$$

It is obvious that the topologies $\tau$ and $\tau^{*}$ satisfy conditions (o), (n) and (d) from 2.1

We shall need in the next theorem the following property:

(r) If $\beta_{n} \in B, x \in X$ and $\varepsilon>0$, then for overy positive integer $p$ there are elements $y$ and $z$ in $X$, such that $x=y+z,\|z\|_{\beta_{i}}^{*}=0$ for $i=1,2, \ldots, p$, and $\|y\| \leqslant \max \left(\|x\|_{\beta_{1}}^{*},\|x\|_{\beta_{2}}^{*}, \ldots,\|x\|_{\beta_{p}}^{*}\right)+\varepsilon$.

3.1.1. THEonem. Suppose that conditions (19) and (c) (seo theorem 2.6.5), or (19) and ( $\mathrm{r}$ ) are satisfied. Then the sets

$$
\bigcap_{i=1}^{\infty}\left[x:\|x\|_{\beta_{i}}^{*} \leqslant \alpha_{i}\right]
$$

where $\beta_{i} \in B$ and $0<\alpha_{i} \rightarrow \infty$, constitute the basis of neighbourhoods of 0 in the mixed topology $\gamma\left[\tau, \tau^{*}\right]$. The mixed topology is atso determined by the pseudonorms

$$
[x]_{\left(\beta_{i}\right),\left(a_{i}\right)}=\sup _{i} \frac{\|x\|_{\beta_{i}}^{*}}{\alpha_{i}}, \quad \beta_{i} \in B, 0<\alpha_{i} \rightarrow \infty .
$$

Proof. It is easy to verify that the class of all sets (20) is a basis of neighbourhoods for 0 in a locally convex linear topology $\tau_{1}$. Let $Z \epsilon \operatorname{Bd}(\tau)$, i. e.

$$
Z \subset[x:\|x\| \leqslant r]
$$

for some $r>0$. Take any neighbourhood of an element $x_{0} \in Z$ in the $\tau_{1} \mid Z$-topology. We can suppose that this neighbourhood is of the form

$$
Z \cap \bigcap_{i=1}^{\infty}\left[x:\left\|x-x_{0}\right\|_{\beta_{i}}^{*} \leqslant \alpha_{i}\right], \quad 0<\alpha_{i} \rightarrow \infty, \quad \beta_{i} \in B .
$$

If $x, x_{0} \in Z$, then, in virtue of $(19),\left\|x-x_{0}\right\|_{\beta_{i}}^{*} \leqslant\left\|x-x_{0}\right\| \leqslant 2 r$. It follows from the condition $\alpha_{i} \rightarrow \infty$ that there exists an integer $i_{0}$ such that $\alpha_{i}>2 v$ for $i \geqslant i_{0}$. We have

$$
Z \frown \bigcap_{i=1}^{i_{0}}\left[x:\left\|x-x_{0}\right\|_{\beta_{i}}^{*} \leqslant \alpha_{i}\right]=Z \frown \bigcap_{i=1}^{\infty}\left[x:\left\|x-x_{0}\right\|_{\beta_{i}}^{*} \leqslant \alpha_{i}\right] .
$$

Hence $\tau^{*}\left|Z \geqslant \tau_{1}\right| Z$, and consequently $\tau_{1} \leqslant \gamma\left[\tau, \tau^{*}\right]$, by 2.2.2.

The proof of the inverse inequality requires the hypothesis that either condition ( $r$ ) or condition (c) is satisfied.

Suppose first that condition ( $\mathbf{r}$ ) holds. Every neighbourhood of 0 in the mixed topology $\gamma\left[\tau, \tau^{*}\right]$ contains a set

$$
U_{0}^{*} \frown \bigcap_{n=1}^{\infty}\left(U_{n}^{*}+n U\right),
$$

where $U=[x:\|x\| \leqslant r], r>0, U_{n}^{*}=\left[x: \max \|x\|_{\beta_{i}}^{*} \leqslant \varepsilon_{n}\right], \beta_{i} \in B, \varepsilon_{n}>0$, $k_{n}<k_{n+1}$ for $n=0,1, \ldots$ Let $\alpha_{i}=\min \left(\varepsilon_{0}, r / 2\right)$ for $1 \leqslant i \leqslant k_{0}$ and $\alpha_{i}=\frac{1}{2} n r$ for $k_{n-1}<i \leqslant k_{n}$. Let $x$ be an arbitrary element of set (20). We have $\|x\|_{\beta_{i}}^{*} \leqslant \alpha_{i} \leqslant \varepsilon_{0}$ for $1 \leqslant i \leqslant k_{0}$ and therefore $x \in D_{0}^{*}$. Let $m$ be a positive integer. It follows from condition ( $\mathrm{r}$ ) (for $p=k_{m}, \varepsilon=\frac{1}{2} m r$ ) that there are elements $y \in X$ and $z \in X$ such that $y+z=x,\|y\| \leqslant \max _{1 \leqslant i \leqslant k_{m}}\|x\|_{\beta_{i}}^{*}+$ $+\frac{1}{2} m r,\|z\|_{\beta_{i}}^{*}=0$ for $1 \leqslant i \leqslant k_{m}$. We have $z \in U_{m}^{*}$ and $\|y\| \leqslant \frac{1}{2} m r+\frac{1}{2} m r$ $=m r$, i. e. $y \in m U$. Consequently $x \in m U+U_{m}^{*}$. The number $m$ being arbitrary, we infer that $x$ belongs to set (21). Therefore set (21) contains set $(20)$ and $\tau_{1} \geqslant \gamma\left[\tau, \tau^{*}\right]$, which, together with the preceding inequality, gives the equality $\tau_{1}=\gamma\left[\tau, \tau^{*}\right]$.

Suppose now that condition (c) is satisfied. We shall use in this case arguments similar to those used in the proof of lemma 1 in [5] (p. 73). Let $U^{\gamma}$ be an open neighbourhood of 0 in the mixed topology. It follows form the equality $\tau^{*}\left|S=\gamma\left[\tau, \tau^{*}\right]\right| S$ that there are $\beta_{1}, \beta_{2}$, $\ldots, \beta_{k_{1}} \in B$, such that

$$
\bigcap_{i=1}^{k_{1}}\left[x:\|x\|_{\beta_{i}}^{*} \leqslant \varepsilon\right] \frown S \subset U^{\gamma} \cap S .
$$


Suppose that there are indices $\beta_{1}, \beta_{2}, \ldots, \beta_{k_{1}}, \beta_{k_{1}+1}, \ldots, \beta_{k_{n}}$ such that (22)

$$
\bigcap_{p=1}^{n} \bigcap_{i=k_{p-1}+1}^{k_{p}}\left[x:\|x\|_{\beta_{i}}^{*} \leqslant \alpha_{i}\right] \cap n S \subset U^{\gamma} \cap n S,
$$

where $\alpha_{i}=\varepsilon$ for $1 \leqslant i \leqslant k_{1}, \alpha_{i}=p-1$ for $k_{p-1}<i \leqslant k_{p}(p=2,3, \ldots)$, $k_{0}=0$. We shall prove that there are $\beta_{k_{n}+1}, \ldots, \beta_{k_{n+1}}$ such that, setting $\alpha_{i}=n$ for $k_{n}<i \leqslant k_{n+1}$, we shall have

$$
\bigcap_{p=1}^{n+1} \bigcap_{i=k_{p-1}+1}^{k_{p}}\left[x:\|x\|_{\beta_{i}}^{*} \leqslant \alpha_{i}\right] \frown(n+1) S \subset U^{\gamma} \frown(n+1) S .
$$

Suppose this be false. Then the set

$$
C_{\gamma_{1}, \gamma_{2}, \ldots, \gamma_{l}}=\bigcap_{x=1}^{n} \bigcap_{i=k_{p-1}+1}^{k_{p}}\left[x:\|x\|_{\beta_{i}}^{*} \leqslant \alpha_{i}\right] \frown \bigcap_{j=1}^{l}\left[x:\|x\|_{\gamma_{j}}^{*} \leqslant n\right]
$$

has, for each finite sequence of indices $\gamma_{1}, \gamma_{2}, \ldots, \gamma_{l} \in B$, a non-void intersection with the set $(n+1) S \backslash U^{\gamma}$. But $\tau^{*}\left|(n+1) S=\gamma\left[\tau, \tau^{*}\right]\right|(n+1) S$ and the set $U^{\gamma}$ is $\gamma\left[\tau, \tau^{*}\right]$-open. Therefore, by condition (c), the set $(n+1) S \backslash U^{\gamma}$ is $\tau^{*}$-compact. Sets $(23)$ are $\tau^{*}$-closed, and it follows from the equality

$$
C_{\gamma_{1}, \gamma_{2}, \ldots, \gamma_{l}} \cap O_{\gamma_{1}^{\prime}, \gamma_{2}^{\prime}, \ldots, \gamma_{l}^{\prime}}=C_{\gamma_{1}, \gamma_{2}, \ldots, \gamma_{l}, \gamma_{1}^{\prime}, \gamma_{2}^{\prime}, \ldots, \nu_{l}^{\prime}}
$$

that the intersection of the members of each finite family of sots (23) has common elements with the $\tau^{*}$-compact set $(n+1) S \backslash U^{\gamma}$. Hence there exists an element

$$
x_{0} \in\left[(n+1) S \backslash U^{\nu}\right] \frown \bigcap_{\gamma_{1}, \ldots, \gamma_{l}} C_{\gamma_{1}, \ldots, \gamma_{l}}
$$

We have $\left\|x_{0}\right\|_{\gamma}^{*} \leqslant n$ for each $\gamma \in B$, and, by condition (19), $\left\|x_{0}\right\| \leqslant n$, i. e. $x_{0} \in n S$. Hence, by (22),

$$
x_{0} \epsilon U^{\gamma} \cap n S \subset U^{\nu} \cap(n+1) S,
$$

in contradiction to (24). Consequently, there exist a sequence of indices $\beta_{i} \in B \quad(i=1,2, \ldots)$, an increasing sequence of positive integers $k_{n}$ $(n=1,2, \ldots)$ and a sequence $0<\alpha_{i} \rightarrow \infty(i=1,2, \ldots)$ such that the inclusion (22) is true for each $n$. We shall have

$$
\bigcap_{i=1}^{\infty}\left[x:\|x\|_{\beta_{i}}^{*} \leqslant \alpha_{i}\right] \frown n \mathcal{S} \subset U^{\gamma}
$$

and, since $n$ is arbitrary,

$$
\bigcap_{i=1}^{\infty}\left[x:\|x\|_{\beta_{i}}^{*} \leqslant \alpha_{i}\right] \subset U^{\gamma} .
$$

Consequently $\tau_{1} \geqslant \gamma\left[\tau, \tau^{*}\right]$. This completes the proof of theorem 3.1.1.

We now give some examples of spaces with mixed topology satisfying the conditions of theorem 3.1.1.

A) Let $X$ be the space $m$ of bounded sequences $x=\left\{t_{i}\right\}$ of real num- bers. The topology $\tau$ is defined by the norm $\|x\|=\sup _{i}\left|t_{i}\right|$, and the topology $\tau^{*}$ is defined by the pseudonorms $\|x\|_{i}^{*}=\left|t_{i}\right|(i=1,2, \ldots)$. It is obvious that conditions (19), (r) and (c) are satisfied. Consequently, the sets $\bigcap_{-1}^{\infty}\left[x:\left|t_{i}\right| \leqslant \alpha_{i}\right]$, where $0<\alpha_{i} \rightarrow \infty$, constitute, by theorem 3.1.1, a basis of neighbourhoods for 0 in the mixed topology.

B) Let $X$ be the space $l$ of sequences $x=\left\{t_{i}\right\}$ of real numbers such that $\sum_{i=1}^{\infty}\left|t_{i}\right|<\infty$. The topology $\tau$ is defined by the norm $\|x\|=\sum_{i=1}^{\infty}\left|t_{i}\right|$, and the topology $\tau^{*}$ is defined by the pseudonorms $\|x\|_{i}^{*}=\left|t_{i}\right|(i=1,2, \ldots)$. The pseudonorms \|\|$_{i}^{*}$ do not satisfy condition (19). We easily observe, however, that the pseudonorms \|\|$_{i}^{*}$ are equivalent to the pseudonorms $[x]_{i}=\sum_{k=1}^{i}\left|t_{k}\right|(i=1,2, \ldots)$, and the pseudonorms []$_{i}$ satisfy condition (19). Conditions (c) and ( $r$ ) are also satisfied and we conclude, by theorem 3.1.1, that the sets $\bigcap_{i=1}^{\infty}\left[x: \sum_{k=1}^{i}\left|t_{k}\right| \leqslant \alpha_{i}\right]$, where $0<\alpha_{i} \rightarrow \infty$, constitute a basis of neighbourhoods for 0 in the mixed topology.

C) Let $T$ be an abstract set, and let $X$ be the space of all bounded, real-valued functions $x=x(t)$ defined on $T$. The topology $\tau$ is defined by the norm $\|x\|=\sup _{t_{\theta} T}|x(t)|$, and the topology $\tau^{*}$ is defined by the pseudonorms $\|x\|_{t}^{*}=\mid \begin{gathered}t_{\epsilon} T \\ |x(t)|\end{gathered}(t \in T)$. Conditions (19), (r) and (c) are satisfied. The sets $\bigcap_{i=1}^{\infty}\left[x:\left|x\left(t_{i}\right)\right| \leqslant \alpha_{i}\right]$, where $t_{i} \in T, 0<\alpha_{i} \rightarrow \infty$, constitute a basis of neighbourhoods for 0 in the mixed topology.

D) Let $T$ be a completely regular Hausdorff space. Let $X$ be the space $C^{*}(T)$ of bounded, real-valued, continuous functions $x=x(t)$ on $T$. Let $\left\{T_{\beta}\right\}_{\beta \in B}$ be a family of (non-necessarily all) compact subsets of $T$ such that $\bigcup_{\beta \in B} T_{\beta}=T$. The topology $\tau$ is defined by the norm $\|x\|=\sup _{t \in T}|x(t)|$, and the topology $\tau^{*}$ is defined by the pseudonorms $\|x\|_{\beta}^{*}=\sup _{t_{\theta} T_{\beta}}|x(t)|$. Condition (19) is obviously satisfied. We shall prove that condition ( $\mathrm{r}$ ) is also satisfied. Let $\beta_{n} \in B, x \in X, \varepsilon>0$, and let $p$ be a positive integer. It is obvious that there exists an open set $G_{p} \subset T$ such that $\bigcup_{i=1}^{p} T_{\beta_{i}} \subset G_{p}$, and

$$
\sup _{t_{e} G_{p}}|x(t)| \leqslant \sup _{t_{\varepsilon} \bigcup_{i=1}^{p} T_{\beta_{i}}}|x(t)|+\varepsilon=\max \left(\|x\|_{\beta_{1}}^{*}, \ldots,\|x\|_{\beta_{p}}^{*}\right)+\varepsilon .
$$

The set $T \backslash G_{p}$ is closed and disjoint with the compact set $F_{p}=\bigcup_{i=1}^{p} T_{\beta_{i}}$. Studia Mathematica $\mathbf{x x}$ 
The space $T$ being completely regular, there exists a bounded, real-valued, continuous function $f(t)$ on $X$ such that $0 \leqslant f(t) \leqslant 1$ for each $t \in T$, $f(t)=0$ for $t \epsilon F_{p}, f(t)=1$ for $t \epsilon T \backslash G_{p}$. Let $y(t)=[1-f(t)] \cdot x(t)$ and $z(t)=f(t) \cdot x(t)$. We have $y \in X, z \in X$ and $x=y+z$. Furthermore, $\|y\|_{\beta}^{*}$ $\leqslant\|x\|_{\beta}^{*}$ and $\|z\|_{\beta}^{*} \leqslant\|x\|_{\beta}^{*}$ for each $\beta \in B$. Since $z(t)=0$ for $t \epsilon F_{p}$, we have $\|z\|_{\beta_{i}}^{*}=0$ for $i=1,2, \ldots, p$. Since $y(t)=0$ for $t \epsilon T \backslash G_{p}$, we have in view of formula (25) $\|y\|=\sup _{t_{\theta} T}|y(t)|=\sup _{t_{\theta} G_{p}}|y(t)| \leqslant \sup _{t_{\theta} G_{p}}|x(t)| \leqslant \max \left(\|x\|_{\beta_{i}}^{*}\right.$, $\left.\ldots,\|x\|_{\beta_{p}}^{*}\right)+\varepsilon$. Consequentiy, the functions $y$ and $\approx t_{\theta}^{t_{\theta} \theta_{p}}$ perties, and condition ( $\mathrm{r}$ ) has been proved.

Applying theorem 3.1.1 we see that the sets

$$
\bigcap_{i=1}^{\infty}\left[x: \sup _{t_{\theta} T_{\beta_{i}}}|x(t)| \leqslant \alpha_{i}\right]
$$

where $\beta_{i} \epsilon B$ and $\dot{0}<\alpha_{i} \rightarrow \infty$, constitute a basis of neighbourhoods for 0 in the mixed topology $\tau^{\gamma}$. In this case the mixed topology is identical with the topology introduced by J. Maŕík [8].

$\mathrm{E})$ Let $X$ be the space conjugate to a normed space $Z$. Let $\tau$ be the strong topology on $X$, defined by the usual norm \|\| of elements of $X$ as functionals, and let $\tau^{*}$ be the weak topology $\sigma(X, Z)$. The topology $\tau^{*}$ may be defined by the pseudonorms $\|x\|_{x}^{*}=|x(z)|$, where $z \in Z,\|z\| \leqslant 1$. The pseudonorms \|\|$_{z}^{*}$ satisfy condition (19). It is well known that condition (c) is also satisfied. By theorem 3.1.1 the sets $\bigcap_{i=1}^{\infty}\left[x:\left|x\left(z_{i}\right)\right| \leqslant \alpha_{i}\right]$, where $z_{i} \in Z,\left\|z_{i}\right\| \leqslant 1,0<\alpha_{i} \rightarrow \infty$, constitute a basis of neighbourhoods for 0 in the topology $\tau^{p}$. We can also say that the sets

$$
\left[x: \sup _{i}\left|x\left(z_{i}\right)\right| \leqslant 1\right] \text {, }
$$

where $z_{i} \in Z,\left\|z_{i}\right\| \rightarrow 0$, constitute a basis of neighbourhoods for 0 in the mixed topology. Consequently, the mixed topology is identical in this case with the topology $\tau_{c}$ of uniform convergence of functionals on the compact subsets of $Z$ ([5], p. 74). In fact, the inequality $\tau_{c} \geqslant \gamma\left[\tau, \tau^{*}\right]$ follows at once from (26). On the other hand, the topology $\tau_{c}$ has property $\left(\mathrm{P}_{1}\right)$ from 2.2, and therefore $\gamma\left[\tau, \tau^{*}\right] \geqslant \tau_{c}$, by 2.2.2.

3.2. We now give two other examples of spaces with mixed topology. The spaces mentioned in F) and $G$ ) do not satisfy the conditions of
theorem 3.1.1.

F) Let $X$ be the space $M$ of measurable, real-valued functions $x(t)$ equivalent to bounded functions on $\langle 0,1\rangle$. The topology $\tau$ is defined by the norm $\|x\|=\sup _{0 \leqslant t \leqslant 1}|x(t)|$, and the topology $\tau^{*}$ is defined by the norm $\|x\|^{*}=\int_{0}^{1}|x(t)| d t$. Conditions (o), (n) and (d) from 2.1 are satisfied.
For each function $x \in X$ and for each $p \geqslant 0$ we write

$$
x_{(p)}(t)=\left\{\begin{array}{lll}
x(t)-p & \text { if } & x(t) \geqslant p, \\
0 & \text { if } & -p \leqslant x(t) \leqslant p, \\
x(t)+p & \text { if } & x(t) \leqslant-p .
\end{array}\right.
$$

The homothetic images (with centre 0 ) of sets

$$
\bigcap_{n=0}^{\infty}\left[x: \int_{0}^{1}\left|x_{(n)}(t)\right| d t \leqslant \varepsilon_{n}\right]
$$

where $\left\{\varepsilon_{n}\right\}$ are arbitrary sequences of positive numbers, constitute a basis of neighbourhoods for 0 in the mixed topology. In fact, set (27) is identical with the set $U_{0}^{*} \frown \bigcap_{n=1}^{\infty}\left(U_{n}^{*}+n U\right)$, where $U_{n}^{*}=\left[x: \int_{0}^{1}|x(t)| d t \leqslant \varepsilon_{n}\right]$ and $U=[x:\|x\| \leqslant 1]$.

G) Let $X$ be the space $\boldsymbol{L}$ of integrable functions on $\langle 0,1\rangle$. The topology $\tau$ is defined by the norm $\|x\|=\int_{0}^{1}|x(t)| d t$ and the topology $\tau^{*}$ is defined by the norm

$$
\|x\|_{1}^{*}=\int_{0}^{1} \frac{|x(t)|}{1+|x(t)|} d t
$$

Conditions (o), (n) and (d) from 2.1 are satisfied. In this case the mixed topology $\tau^{\gamma}$ is not locally convex. Alexiewicz ([2], p. 54) has shown that there are no non-trivial linear functionals on the space $\left\langle X, \tau^{\gamma}\right\rangle$.

\section{References}

[1] A. Alexiewicz, On sequences of operations (II), Studia Math. 11 (1950), p. 200-236.

[2] - On the two-norm convergence, ibidem 14 (1954), p. 49-56.

[3] A. Alexiewicz and Z. Semadeni, A generalization of two-norm spaces, Bull. Pol. Acad. Sci. 6 (1958), p. $135-139$.

[4] - Linear functionals on two-norm spaces, Studia Math. 17 (1958), p. 121-140.

[5] N. Bourbaki, Éléments de mathématique, Livre $V:$ Espaces vectoriels topologiques, Chapters III-V, Act. sci. et ind., no.1229, Paris 1955.

[6] J. A. Dieudonné, Recent developments in the theory of locally convex vector spaces, Bull. Amer. Math. Soc. 59 (1953), p. 495-512.

[7] G. Fichtenholz, Sur les fonctionnelles linéaires continues au sens généralisé, Mat. Sbornik 4 (1938), p. 193-214.

[8] J. Mařík, Les fonctionnelles sur l'ensemble des fonctions continues bornées, définies dans un ensemble topologique, Studia Math. 16 (1957), p. 86-94. 
[9] W. Orlicz, Linear operations in Saks spaces (I), ibidem 11 (1950), p. $237-272$.

[10] - Linear "operations in Sals spaces (II), ibidem 15 (1956), p. 1-25.

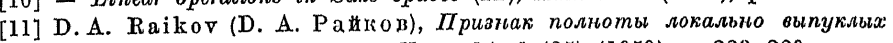
пространств, Успехи Математических Нау'к 14, 1 (85) (1959), p. 223-229.

[12] A. Wiweger, A topologisation of Salss spaces, Bull. Pol. Acad. Sci. 5 (1957), p. $773-777$.

INSTYTUT MATEMATYCZNX POLSKIXIJ AKADKMII NAUK

MATHAMATICAL INSTITUTE OF THE POLTSE ACADTEMY OF SCMENCRS

\section{Extinguishing a class of functions}

Iby

Reşu par la Rédaction le 29. 3. 1960

H. FAST (Jerusalem) and K. URBANIK (Wrocław)

Let $E$ be a set of real positive numbers. By $L(E)$ we shall denote the family of all intervals of the form

$$
I=\{(x, y): \alpha x+y=t, x \geqslant 0, y \geqslant 0\},
$$

where $a \epsilon E$ and $0<t<\infty$. A complex-valued continuous function $\varphi$ of two variables defined on the first quadrant is said to be extinguished by the set $E$ if $\int \varphi(x, y) d s=0$ for any interval $I \epsilon L(E)$. It is well known ([2], p. 63) that

(*) The unique function extinguished by the right half-line is the function identically equal to 0 .

Let $s q_{n}$ denote the class of all complex-valued functions $\varphi$ of two variables defined on the first quadrant and having the representation

$$
\varphi(x, y)=\sum_{j=1}^{n} f_{j}(x) g_{j}(y),
$$

where all the functions $f_{1}, f_{2}, \ldots, f_{n}, g_{1}, g_{2}, \ldots, g_{n}$ are continuous on the right half-line. By $\mathfrak{E}_{n}$ we shall denote the class of all sets $E$ of positive numbers such that the unique function belonging to $\mathscr{A}_{n}$ and extinguished by $E$ is the function identically equal to 0. From Titchmarsh's Theorem on convolution $\left([3]\right.$, p. 327) it follows that all one-point sets belong to $\mathfrak{E}_{1}$. Indeed, if a function $\varphi$ is extinguished by a set $\{\alpha\}$ and $\varphi(x, y)=f(x) g(y)$, then we have the equality

$$
\int_{a x+y=t} f(x) g(y) d s=0 \quad(t>0) .
$$

Hence for any positive $t$ we get the equality

$$
\int_{0}^{t} f(x) g(\alpha(t-x)) d x=0,
$$

